\title{
Tau Reconstruction and Identification Performance at ATLAS
}

\author{
Björn Gosdzik \\ on behalf of the ATLAS Collaboration \\ Deutsches Elektronen-Synchrotron, Notkestrasse 85, D-22607 Hamburg, GERMANY
}

\begin{abstract}
For many signals in the Standard Model including the Higgs boson, and for new physics like Supersymmetry, $\tau$ leptons represent an important signature. This work shows the performance of the ATLAS $\tau$ reconstruction and identification algorithms. It will present a set of studies based on data taken in 2010 at a center-of-mass energy of $\sqrt{s}=7 \mathrm{TeV}$. We measured some of the basic input quantities used for these identification methods from selected reconstructed $\tau$ candidates and compared the results to the prediction of different Monte Carlo simulation models. For early data taking a cut-based identification method will be used. We also measured the background efficiency for the cut-based $\tau$ identification.
\end{abstract}

\section{Introduction}

In the Standard Model, a large number of $\tau$ leptons is expected from the decay of $Z$ and $W$ bosons with $100 \mathrm{pb}^{-1}$ of data. $\tau$ leptons also play an important role in searches for new phenoma like the Standard Model Higgs boson, MSSM Higgs bosons and SUSY with large $\tan \beta$ since $\tau$ leptons can differentiate between SUSY models based on polarization information. They have a mass of $\mathrm{m}_{\tau}=1.78 \mathrm{GeV}$ and decay $\approx 65 \%$ of the time hadronically.

$\tau$ leptons in ATLAS typically have a collimated calorimetric cluster, 1 or 3 charged decay products and a displaced secondary vertex in the case of 3-prong decays.

\section{Reconstruction and Identification}
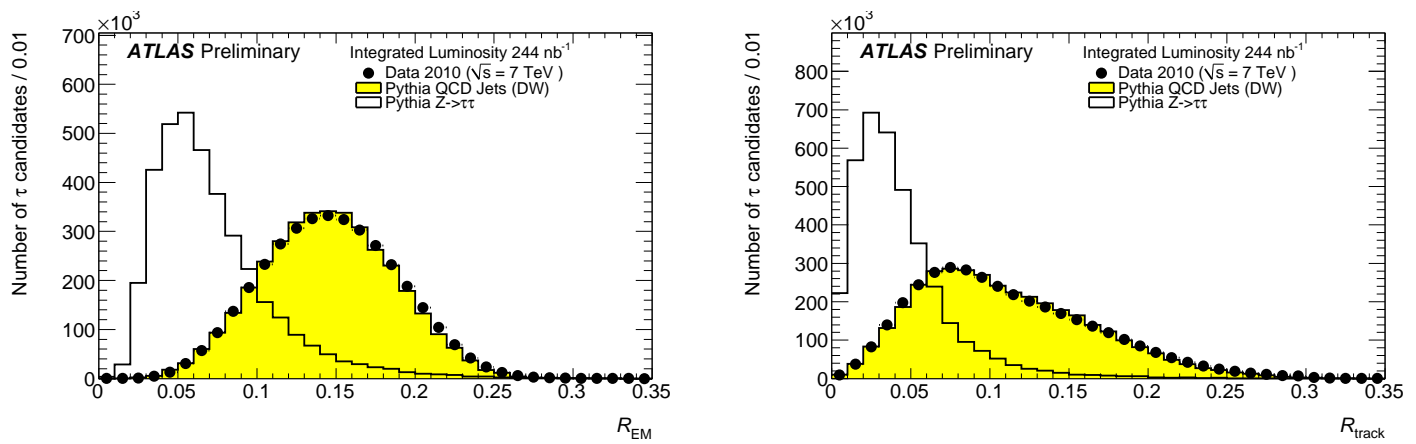

Figure 1: EM radius (left) and track radius (right), see section 3 for definitions [2].

The studies are based on data collected with the ATLAS detector [1] at a center-of-mass energy of $\sqrt{\mathrm{s}}=7 \mathrm{TeV}$ and correspond to an integrated luminosity of approximately $\mathcal{L}=244 \mathrm{nb}^{-1}$. Dedicated cuts on the data, haven been applied to select events with back-to-back jets and to enrich the sample with fake $\tau$ candidates from QCD processes that form the primary background [2] in searches with $\tau$ lepton final states.

To compare the distribution of the variables used for the $\tau$ reconstruction and identification we used predictions from QCD jets Monte Carlo (MC) samples, generated with the Pythia DW tune [3]. The reconstruction of hadronically decaying $\tau$ leptons starts from either calorimeter or track seeds:

- Track-seeded candidates start with a seeding track of $p_{\mathrm{T}}>6 \mathrm{GeV},|\eta|<2.5$, and satisfy quality criteria on the impact parameter with respect to the interaction vertex $\left(\left|d_{0}\right|<2 \mathrm{~mm}\right.$ and $\left.\left|z_{0}\right| \times \sin \theta<10 \mathrm{~mm}\right)$. 
- Calorimeter-seeded candidates consist of calorimeter jets reconstructed with the anti-Kt algorithm (using a distance parameter $D=0.4)$ starting from topological clusters with a calibrated $E_{\mathrm{T}}>10 \mathrm{GeV}$ and $|\eta|<2.5$.

- Candidates are labeled double-seeded when a seed track and a seed jet are within a distance of $\Delta \mathrm{R}=\sqrt{(\Delta \eta)^{2}+(\Delta \phi)^{2}}<0.2$.

Seven variables are currently used as inputs for the identification algorithms to distinguish $\tau$ leptons from QCD jets. The variables electromagnetic (EM) radius and track radius are shown in Fig. 1 .

\section{Background Rejection in QCD events}
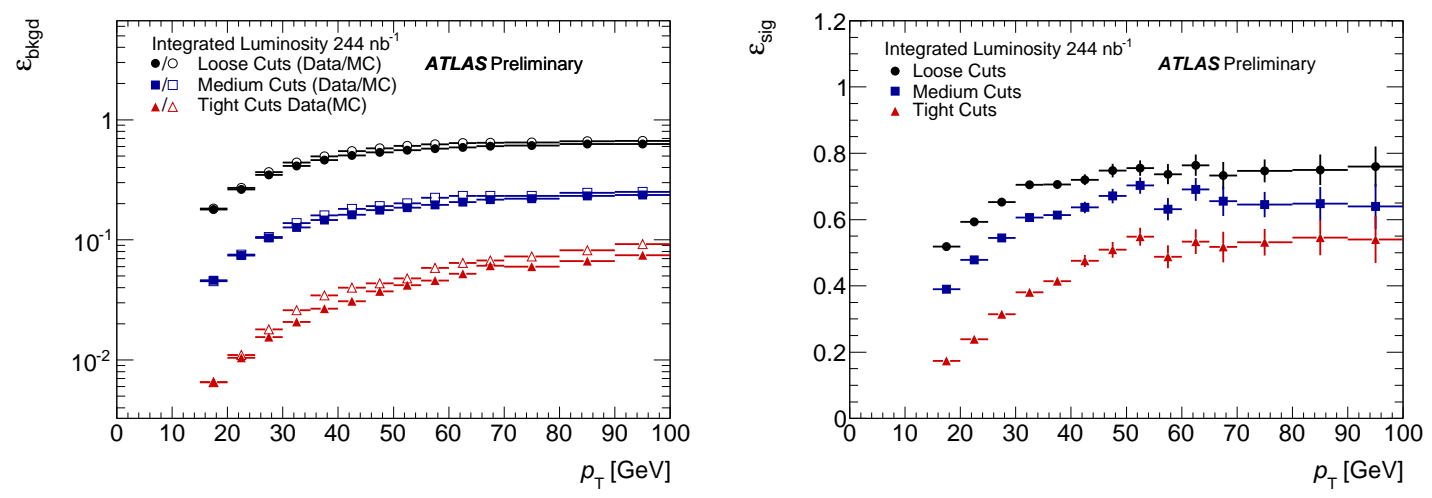

Figure 2: Background efficiency from Data/MC as a function of the reconstructed $p_{\mathrm{T}}$ of the $\tau$ candidate (left); signal efficiency from $Z \rightarrow \tau \tau \mathrm{MC}$ as a function of the reconstructed $p_{\mathrm{T}}$ (right) [2].

To identify $\tau$ leptons after the reconstruction, three independent identification (ID) algorithms were studied: simple cuts, boosted decision trees (BDT) and a projective likelihood (LL).

The cut-based ID uses three variables: $R_{\mathrm{EM}}=\frac{\sum_{i}^{\Delta R_{i}<0.4} E_{\mathrm{T}, i}^{\mathrm{EM}} \Delta R_{i}}{\sum_{i}^{\Delta R_{i}<0.4} E_{\mathrm{T}, i}^{\mathrm{EM}}}, R_{\text {track }}=\frac{\sum_{i}^{\Delta R_{i}<0.2} p_{\mathrm{T}, i} \Delta R_{i}}{\sum_{i}^{\Delta R_{i}<0.2} p_{\mathrm{T}, i}}$ and $f_{\mathrm{trk}, 1}=\frac{p_{\mathrm{T}, 1}^{\mathrm{track}}}{p_{\mathrm{T}}^{\tau, v i s}}$. Three selections corresponding to signal efficiencies of $30 \%$ (tight), $50 \%$ (medium), and $60 \%$ (loose) are optimized to maximize the rejection of QCD jets. The background efficiency for all three selections is shown in Table 1 The background efficiency $\varepsilon_{\text {bkgd }}^{\prime}$ requires $n_{\text {track }}=1$ or $n_{\text {track }}=3$. Figure 2 shows the background efficiency from Data/MC and signal efficiency from $Z \rightarrow \tau \tau$ MC for the cut-based ID. Figure 3 (left) shows the BDT jet score and Fig. 3 (right) the likelihood score. The number of $\tau$ candidates in the MC samples is normalized to the number of $\tau$ candidates in the data. Very good agreement between the data and the prediction of QCD MC is observed.

\begin{tabular}{l|cc|cc}
\hline \hline Selection & $\varepsilon_{\text {bkgd }}($ data $)$ & $\varepsilon_{\text {bkgd }}(\mathrm{MC})$ & $\varepsilon_{\text {bkgd }}^{\prime}($ data $)$ & $\varepsilon_{\text {bkgd }}^{\prime}(\mathrm{MC})$ \\
\hline loose & $(3.2 \pm 0.2) \times 10^{-1}$ & $3.4 \times 10^{-1}$ & $(9.4 \pm 0.6) \times 10^{-2}$ & $10 \times 10^{-2}$ \\
medium & $(9.5 \pm 1.0) \times 10^{-2}$ & $9.9 \times 10^{-2}$ & $(3.1 \pm 0.4) \times 10^{-2}$ & $3.3 \times 10^{-2}$ \\
tight & $(1.6 \pm 0.3) \times 10^{-2}$ & $1.9 \times 10^{-2}$ & $(5.6 \pm 0.9) \times 10^{-3}$ & $6.8 \times 10^{-3}$ \\
\hline \hline
\end{tabular}

Table I: Background efficiencies for loose, medium, and tight selection cuts. The measured background efficiencies in data are compared to the MC DW tune prediction [2].

Two effects contribute to systematic uncertainties:

- The transverse momentum calibration: Two calibration schemes have been compared, a global cell energydensity weighting $(\mathrm{GCW})$ and a simple $p_{\mathrm{T}}$ and $\eta$ dependent calibration (EM+JES). The ratio of the background efficiency for both calibration schemes as function of $p_{\mathrm{T}}$ is shown in Fig. 4 (left). 

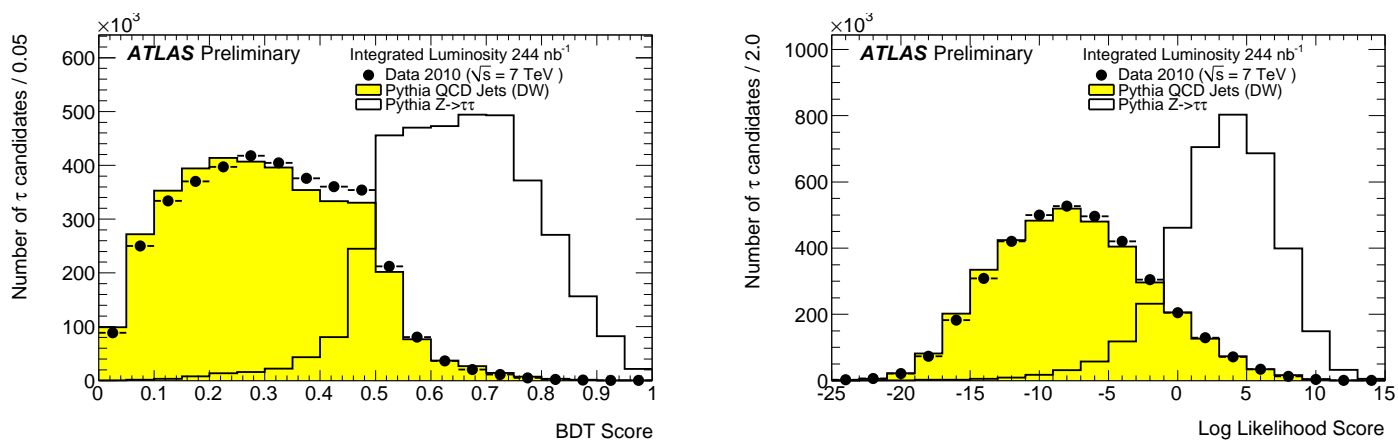

Figure 3: BDT jet score (left) and LL score (right) for $\tau$ candidates in data and MC samples [2].

- The pile-up effect: During the data taking period, the beam intensity has increased significantly. The number of vertices $n_{v t x}$ is highly correlated with pile-up activity. The background efficiency as function of $n_{v t x}$ is shown in Fig. 4 (right).
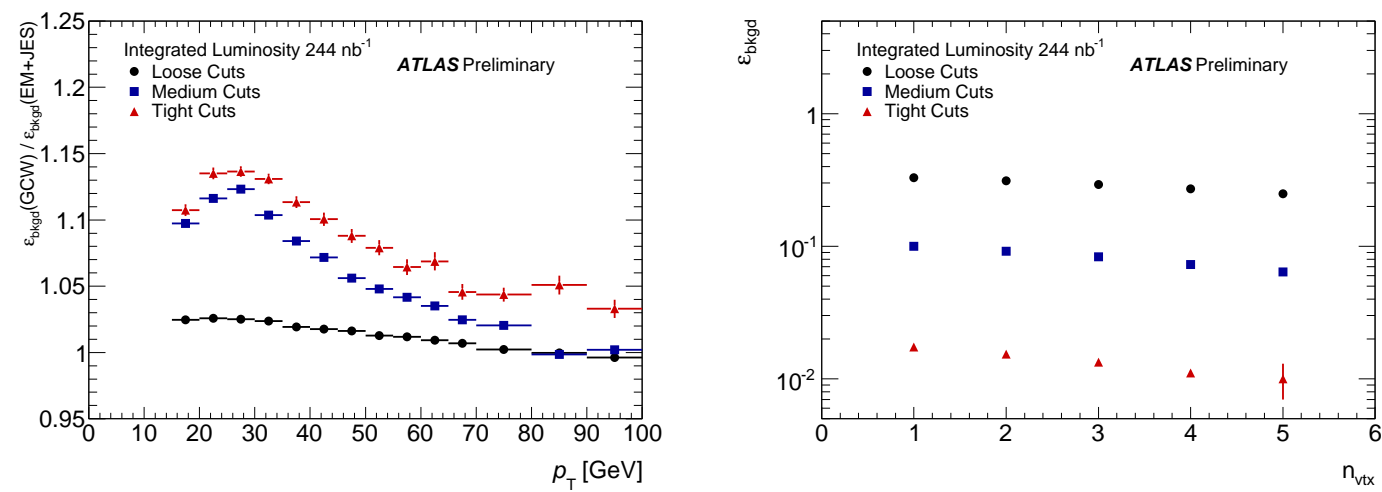

Figure 4: Ratio of background efficiencies using EM+JES and GCW calibration as a function of $p_{\mathrm{T}}$ (left); background efficiencies as a function of $n_{v t x}$ (right) [2].

\section{Conclusion}

All variables used in the $\tau$ ID algorithm are well described by MC predictions and show good separation power between $\tau$ leptons and fake $\tau$ candidates from QCD jets. Altogether the commissioning of the tauID was successful.

\section{Acknowledgments}

Work supported by the Helmholtz Young Investigators Group VH-NG-303.

\section{References}

1 The ATLAS Collaboration, The ATLAS Experiment at the CERN Large Hadron Collider, JINST 3 (2008) S08003.

2 The ATLAS Collaboration, Tau Reconstruction and Identification Performance at ATLAS, ATLAS-CONF-2010086.

3 TeV4LHC QCD Working Group, Tevatron-for-LHC Report of the QCD Working Group, arXiv:hep-ph/0610012v1.

Proceedings of HCP2010 - Toronto 